\title{
Accuracy of Preoperative Imaging in Detecting Nodal Extracapsular Spread in Oral Cavity Squamous Cell Carcinoma
}

\author{
A.H. Aiken, S. Poliashenko, J.J. Beitler, A.Y. Chen, K.L. Baugnon, A.S. Corey, K.R. Magliocca, and DP.A. Hudgins
}

\begin{abstract}
BACKGROUND AND PURPOSE: The increasing impact of diagnosing extracapsular spread by using imaging, especially in patients with oropharyngeal squamous cell carcinoma, highlights the need to rigorously evaluate the diagnostic accuracy of imaging. Previous analysis suggested $62.5 \%-80.9 \%$ sensitivity and $60 \%-72.7 \%$ specificity. Our goals were to evaluate the accuracy of imaging in diagnosing extracapsular spread in a cohort of patients with oral cavity squamous cell carcinoma (pathologic confirmation of extracapsular spread routinely available), as a proxy for oropharyngeal squamous cell carcinoma, and to independently assess the reliability of imaging features (radiographic lymph node necrosis, irregular borders/stranding, gross invasion, and/or node size) in predicting pathologically proven extracapsular spread.
\end{abstract}

MATERIALS AND METHODS: One hundred eleven consecutive patients with untreated oral cavity squamous cell carcinoma and available preoperative imaging and subsequent lymph node dissection were studied. Two neuroradiologists blinded to pathologically proven extracapsular spread status and previous radiology reports independently reviewed all images to evaluate the largest suspicious lymph node along the expected drainage pathway. Radiologic results were correlated with pathologic results from the neck dissections.

RESULTS: Of 111 patients, 29 had radiographically determined extracapsular spread. Pathologic examination revealed that 28 of 111 (25\%) had pathologically proven extracapsular spread. Imaging sensitivity and specificity for extracapsular spread were $68 \%$ and $88 \%$, respectively. Radiographs were positive for lymph node necrosis in $84 \%$ of the patients in the pathology-proven extracapsular spread group and negative in only $7 \%$ of those in the pathologically proven extracapsular spread-negative group. On logistic regression analysis, necrosis $(P=.001)$, irregular borders $(P=.055)$, and gross invasion $(P=.068)$ were independently correlated with pathologically proven extracapsular spread.

CONCLUSIONS: Although the specificity of cross-sectional imaging for extracapsular spread was high, the sensitivity was low. Combined logistic regression analysis found that the presence of necrosis was the best radiologic predictor of pathologically proven extracapsular spread, and irregular borders and gross invasion were nearly independently significant.

ABBREVIATIONS: $\mathrm{ECS}=$ extracapsular spread; OCSCC = oral cavity squamous cell carcinoma; OPSCC = oropharyngeal squamous cell carcinoma; $\mathrm{pECS}=$ pathologically proven extracapsular spread

$\mathbf{T}$ he presence of pathologically proven extracapsular spread (pECS) in squamous cell carcinoma of the head and neck portends a poor prognosis with decreased 5-year overall survival rates and increased rates of locoregional recurrences and distant

\footnotetext{
Received March 3, 2014; accepted after revision March 3, 2015.

From the Departments of Radiology and Imaging Sciences (J.J.B., A.H.A., A.S.C., K.L.B., P.A.H.), Otolaryngology Head and Neck Surgery (A.Y.C., J.J.B.), Radiation Oncology (J.J.B.), Hematology and Medical Oncology, and Pathology (K.R.M.), Emory University School of Medicine (S.P.), Atlanta, Georgia.

Paper previously presented at: Annual Meeting of the American Society of Neuroradiology, May 18, 2013, 2013; San Diego, California.

Please address correspondence to A.H. Aiken, MD, Emory University Hospital, 1364 Clifton Rd NE, Atlanta GA 30322; e-mail: ashley.aiken@emoryhealthcare.org

http://dx.doi.org/10.3174/ajnr.A4372
}

metastases. ${ }^{1,2}$ In a large 10-year multicohort study, Shaw et al ${ }^{3}$ found that extracapsular spread (ECS) in patients with oral cavity squamous cell carcinoma (OCSCC) doubled the local recurrence rate, doubled the distant metastasis rate, and tripled the incidence of regional recurrence. However, recent literature has debated whether ECS affects disease-specific survival in patients with p16positive or p16-negative oropharyngeal squamous cell carcinoma (OPSCC). ${ }^{4}$ Radiographically determined ECS has been shown to be an independent prognosticator for poor distant control and survival, ${ }^{5}$ but although CT and MR imaging may suggest the radiologically visible presence of macroscopic ECS, the criterion standard for diagnosing ECS in patients with head and neck cancer has been pathologic evaluation of a neck-dissection specimen. Distinct from OCSCC, oropharyngeal cancers (eg, OPSCC) 
are routinely and successfully treated with radiation therapy alone for early disease and chemoradiation for more advanced disease. ${ }^{6}$ Technical advances have resulted in a renewed interest in transoral robotic surgery for the treatment of early disease. ${ }^{7}$ Some patients with surgically treated early OPSCC are spared the need for adjuvant radiation. Conversely, because a combination of European Organisation for Research and Treatment of Cancer and Radiation Therapy Oncology Group studies showed survival advantages when chemotherapy was added to routine postoperative radiation in patients who had either unsatisfactory margins or $\mathrm{ECS},{ }^{8}$ many clinicians would not offer surgery for patients with oropharyngeal cancer if they knew preoperatively that pECS was present. Therefore, to spare the patients trimodality treatment, especially in the human papillomavirus era, patients with oropharyngeal cancer with ECS on pretherapy imaging are generally treated with concurrent chemoradiation, and surgery is reserved for possible salvage. In light of these considerations, radiographically determined ECS is crucial for the upfront management of OPSCC.

The crucial role of preoperative imaging in this particular scenario prompted our investigation of the accuracy of imaging for diagnosing ECS. Although our clinical question arose from patients with OPSCC, we used patients with OCSCC in this study as a proxy cohort, because elective neck dissection provides a pECS status for nearly all patients.

The sensitivity and specificity for radiographically determined ECS have been reported to range from $62.5 \%$ to $80.9 \%$ and from $60 \%$ to $93 \%$, respectively. ${ }^{9-14}$ A small study in 17 patients by Zoumalan et $\mathrm{al}^{15}$ found that lymph node necrosis was a good predictor of ECS. Classical imaging features of ECS include spiculated or irregular node borders, loss of the adjacent fat or muscle planes, and frank infiltration into structures adjacent to the lymph node. ${ }^{16}$ Our study objectives were to evaluate the accuracy of cross-sectional contrast-enhanced CT imaging for diagnosing ECS in patients with OCSCC (as a proxy for those with OPSCC) and to independently assess the reliability of imaging features (radiographically determined lymph node necrosis, irregular borders/stranding, gross invasion, and/or node size) for predicting pECS.

\section{MATERIALS AND METHODS \\ Inclusion Criteria}

After institutional review board approval for this retrospective review, the imaging data warehouse at our institution was queried for previously untreated patients with International Classification of Diseases, Ninth Revision, codes for OCSCC subsites between 2007 and 2012. This search yielded 229 patients. Subsites of OCSCC included in the study were the oral tongue, retromolar trigone, buccal mucosa, and gingival regions. Inclusion criteria were having had 1) a neck dissection at our institution, 2) preoperative contrast-enhanced CT, and 3) pathologic review specifically for ECS.

From the 229 patients identified in this search, 165 who underwent subsequent neck dissection at our institution were included. Fifty-four patients were excluded because preoperative images were not available $(n=43)$, they underwent only MR imaging $(n=8)$, or their CT was performed without contrast $(n=3)$. Therefore, 111 patients with preoperative CT imaging and who had a neck dissection at our institution and pathologic evaluation for ECS were included in this study.

\section{Pathology Review}

The diagnosis of squamous cell carcinoma, pathologically determined lymph node stage, lymph node size, and presence of ECS were recorded from original pathology reports in the electronic medical record. The histologic criterion for extracapsular spread in a metastatic lymph node was defined as carcinoma invading beyond the nodal capsule. The measured extent of carcinoma beyond the lymph node capsule (micro-ECS vs macro-ECS) was not a routine practice during this timeframe. This cohort included a mixture of radiographically determined positive and negative necks and pathologically determined positive and negative necks. All neck dissections for OCSCC in the study time range that met inclusion criteria were included.

\section{Imaging Methods}

Patients underwent imaging on one of several commercially available CT systems with multidetector capability ranging from 8 to 64 channels. Onsite imaging CT studies were performed on a LightSpeed VCT 64-section, Discovery HD 750, Discovery 16section, or BrightSpeed 16-section scanner (all from GE Healthcare, Milwaukee, Wisconsin) or Somatom Definition 64-slice and Somatom Emotion 16-slice CT scanners (Siemens, Erlangen, Germany). Our split-bolus technique used a total of $110 \mathrm{~mL}$ of intravenous iopamidol (Isovue-370; Bracco, Princeton, New Jersey); $55 \mathrm{~mL}$ was injected first at a rate of $2.5 \mathrm{~mL} / \mathrm{s}$, followed by a 40 -second delay, and then another $55 \mathrm{~mL}$ was added at the same rate, with a total scan delay of 90 seconds, including a precontrast and postcontrast saline bolus. We acquired contiguous axial images from the skull base through the mediastinum with the following settings: section thickness, $1.25 \mathrm{~mm}$; pitch, 0.984:1; gantry rotation time, 0.7 second; FOV, $25 \mathrm{~cm} ; 120 \mathrm{kVP}$; and Smart mA with a noise index of 13.78. Reformatted images with $2.5-\mathrm{mm}$ section thicknesses in the axial planes and 3-mm sagittal and coronal reformations were sent to a PACS.

\section{Image Review}

Two Certificate of Added Qualification-certified neuroradiologists with a focus on head and neck imaging (A.H.A. and P.A.H., who have 8 and 29 years of experience, respectively) reviewed all 111 cases. The reviewers were blinded to the original reports, final pathology, and the other reviewer's conclusions. For each case, they recorded a dichotomous interpretation for the following features of the largest suspicious ipsilateral node along the expected drainage pathway: 1) mild perinodal stranding or irregular node borders, 2) necrosis, 3) gross invasion, and 4) overall impression of macroscopic ECS. Evidence of ECS was defined as irregular node borders, fat stranding, and/or invasion of adjacent structures (Fig 1). When there was disagreement between the 2 reviewers, a consensus read without knowledge of pECS status was performed.

In addition to the blinded review, each of the 111 cases was interpreted initially by 1 of 4 Certificate of Added Qualificationcertified and dedicated head and neck radiologists in our practice 

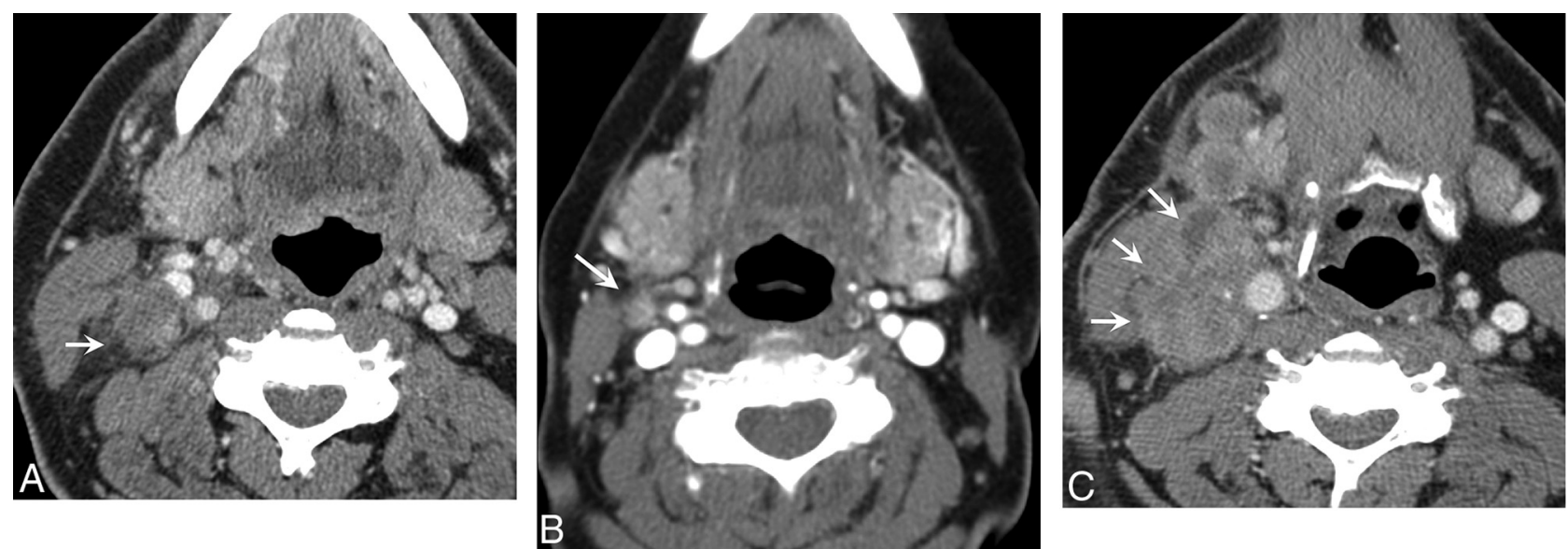

FIG 1. A, A right level IIA lymph node (arrow) in a patient with OCSCC. This rounded low-attenuation node has irregular borders and fat stranding. Imaging prospectively diagnosed ECS, which was confirmed with pathologic examination (true-positive result). B, A right level IIA lymph node (arrow) in a patient with OCSCC. This small 1-cm lymph node shows subtle fat stranding. Imaging prospectively diagnosed ECS, which was confirmed with pathologic examination (true-positive result). C, A right level IIA lymph node (arrows) in a patient with OCSCC. This large necrotic node demonstrates invasion of the adjacent sternocleidomastoid muscle. Imaging prospectively diagnosed ECS, which was confirmed with pathologic examination (true-positive result).

(each with 6, 8, 15, or 29 years' experience). Initial reports included the radiologic staging of lymph nodes, presence of radiographically determined ECS, size of the nodal disease, and lymph node necrosis. Node size was measured in the longest axis in the axial plane and categorized into one of the following groups: $0-1$, $1-2,2-3$, or $>3 \mathrm{~cm}$.

\section{Statistical Analysis}

A multivariate logistic regression analysis was performed, and the sensitivity, specificity, accuracy, positive predictive value, and negative predictive value were calculated. A Cohen weighted $k$ statistic was calculated to evaluate interobserver agreement. Observer agreement was categorized as poor $(<0.20)$, fair $(0.20-$ $0.39)$, moderate $(0.40-0.59)$, good $(0.60-0.79)$, or excellent $(\geq 0.80)$.

\section{RESULTS}

Our population included 111 patients with OCSCC who underwent primary resection and neck dissection at our institution within a 5-year period.

Interobserver analysis revealed almost perfect interobserver agreement between the readers for overall impression of ECS $(\kappa=$ 0.86; $P<.001)$ and for stranding/irregular borders around a lymph node $(\kappa=0.835 ; P<.001)$. Radiographically determined ECS was noted in 29 of the 111 cases, and 19 of those 29 were true-positive results. There were 9 false-negative results. Pathologically proven ECS was confirmed in 28 of 111 cases. The readers had only 6 of 111 discrepancies for ECS, with each reader's conclusion being validated pathologically in 3 of 6 cases. Therefore, the readers had the same overall sensitivity and specificity results. For the review, the imaging diagnosis of ECS had a sensitivity of $68 \%$, a specificity of $88 \%$, a positive predictive value of $66 \%$, and a negative predictive value of $89 \%$.

Radiographically determined lymph node necrosis was present in 27 of 111 patients, and 21 of these 27 lymph nodes were truly positive for ECS. Radiographically determined lymph node necrosis, therefore, had a sensitivity of $66 \%$ and a specificity of
93\% for predicting pECS. Radiographically determined lymph node necrosis was present in $84 \%$ of nodes with pathologyproven ECS but only $7 \%$ of nodes without ECS ( 6 of 86 ). There was a strong association between radiologically determined lymph node necrosis and pECS $(P<.001)$ according to the Fisher exact test.

Radiographic evidence of gross invasion of surrounding structures, such as the sternocleidomastoid muscle, was noted in 8 of 111 cases, and pECS was present in all 8 of these cases (specificity, $100 \%)$. As expected, the sensitivity of gross invasion was low (34\%).

Radiographically determined perinodal stranding had a sensitivity of $63 \%$ and a specificity of $89 \%$ for predicting ECS, very similar to the overall imaging sensitivity and specificity rates; stranding usually prompts a positive overall impression for radiographically determined ECS.

A logistic regression analysis was performed for each of the reviewers to determine which radiologic factors were most predictive of ECS (Table). Imaging necrosis had the highest classification accuracy (ie, it was the most predictive of pECS $[P=.001$ for the combined analysis $])$. Stranding $(P=.055)$ and gross invasion $(P=.068)$ were also marginally significant. Therefore, all 3 factors were nearly independently significant.

From the original reports, radiographically determined ECS was reported in only 17 cases (compared with 29 cases in the blind review). The original reports' diagnosis of ECS had a sensitivity of $46 \%$, a specificity of $95 \%$, a positive predictive value of $76 \%$, and a negative predictive value of $84 \%$. The interobserver variability between the original report and the blinded review showed good or substantial agreement $(\kappa=0.676 ; P<.001)$.

Two-sample $t$ tests were performed to compare lymph node size between the pathologically positive and negative ECS groups. Continuous variables for those in the ranges of $0-1,1-2,2-3$, and $>3 \mathrm{~cm}$ were evaluated. For all of the groups, the $P$ value was .20, suggesting that we could not find a significant association between size and ECS, primarily because $100 \%$ (4 of 4) of the small- 
Logistic Regression Analysis for individual readers and combined analysis

\begin{tabular}{lcccc}
\hline Reader No. and Parameter & Estimate & Standard Error & $\boldsymbol{z}$ Value & $\operatorname{Pr}(>|z|)$ \\
\hline 1 (A.H.A.) & & & & \\
$\quad$ (Intercept) & -2.045 & 0.344 & -5.948 & $<.001$ \\
Stranding & 1.072 & 0.741 & 1.447 & .148 \\
Necrosis & 1.714 & 0.78 & 2.198 & .028 \\
Gross invasion & 1.553 & 1.208 & 1.285 & .199 \\
2 (P.A.H.) & & & & \\
(Intercept) & -2.229 & 0.370 & -6.016 & $<.001$ \\
Stranding & 0.875 & 0.764 & 1.145 & .252 \\
Necrosis & 1.902 & 0.702 & 2.710 & .007 \\
Gross invasion & 1.306 & 0.975 & 1.339 & .180 \\
1 and 2 (A.H.A. and P.A.H. & & & & \\
[combined analysis]) & & & & \\
(Intercept) & -2.129 & 0.251 & -8.493 & 0 \\
Stranding & 1.006 & 0.525 & 1.917 & .055 \\
Necrosis & 1.78 & 0.515 & 3.457 & .001 \\
Gross invasion & 1.358 & 0.744 & 1.824 & .068 \\
\hline
\end{tabular}

Note: $-\operatorname{Pr}(>|z|)$ indicates a $P$ value from a $z$ score.

est group of lymph nodes $(<1 \mathrm{~cm})$ had ECS. It should be noted that these were false-negative radiographic results. However, the results of an evaluation of the remaining individual groups suggested a trend. The rates of pathologically proven ECS among the other groups was $60 \%$ in the 1 - to 2 -cm group, $83 \%$ in the 2 - to $3-\mathrm{cm}$ group, and $100 \%$ in the $>3-\mathrm{cm}$ group.

\section{DISCUSSION}

It became clear during our multidisciplinary tumor boards that our clinicians were increasingly relying on the imaging diagnosis of ECS to make major treatment decisions in patients with OPSCC. Escalating therapy to a multimodal approach involves significant morbidity and thus must be appropriately justified. Specifically, our clinicians were deciding against transoral robotic surgery in patients with preoperative radiographic evidence of ECS to avoid trimodality therapy for the patients. Because our institution did not have an adequate sample size to test the accuracy of imaging in diagnosing ECS in patients with OPSCC, we chose to evaluate its accuracy in a welldefined cohort of patients with OCSCC. Our primary objective was to determine the accuracy of imaging in diagnosing ECS so that our tumor board could best plan the treatment of patients with OPSCC, assuming that it is reasonable to extrapolate results based on OCSCC and apply them to OPSCC.

If treatment decisions are going to potentially be based on imaging detection of ECS, then the accuracy and limitations of imaging results must be taken into account. When we reviewed the literature, we found wide variation in the accuracy of imaging, mixed cohorts, and conflicting data. Furthermore, at least for oropharyngeal cancer, the biology has changed; most of our oropharyngeal cancers are related to human papillomavirus rather than to smoking or drinking alcohol. ${ }^{17}$ The cystic appearance of many human papillomavirus 16 positive oropharyngeal node metastases may complicate the radiologic evaluation of necrosis. Another important consideration is whether to err on the side of being overly sensitive or of being overly specific. ${ }^{18}$

Recently, Prabhu et $\mathrm{al}^{19}$ examined 432 patients undergoing neck dissections for head and neck cancer. The sensitivity and specificity for radiographically determined ECS were reported to be $43 \%$ and $97.7 \%$, respectively. The sensitivity was lower and specificity much higher than previously reported. One possibility for the lower sensi- tivity of radiographically determined ECS in their study may have been the criteria for radiographically positive and negative ECS. When ECS status was not mentioned in the initial radiology report, it was recorded as radiographically negative for ECS. The preoperative images were not blindly reviewed, because the authors' purpose was to assess the predictive value of the original diagnostic interpretation. Because many of the study patients had OCSCC and the radiographic diagnosis of ECS would have been less critical and had little impact on the decision for neck dissection, it may not have always been reported initially by the neuroradiologist, falsely depressing sensitivity. Our study addressed this potential pitfall with a blinded review for all cases, in addition to the initial report, to validate these preliminary results.

In a second recent study, Chai et $\mathrm{al}^{20}$ concluded that "radiologic findings suggestive of ECS should not be relied on for treatment planning in squamous cell carcinoma of the head and neck." Chai et $\mathrm{al}^{20}$ used a 5-point scale for the evaluation of radiographically determined ECS, included a wider range of primary tumors, and had 2 different observers with specificities of $54 \%$ and $84 \%$, respectively. Our study aimed to limit a few of these variables by restricting the cohort to a single primary tumor for which surgical resection of the primary and cervical lymphatics is the standard initial treatment and by reporting "yes or no" for radiographically determined ECS on the basis of specific imaging features.

Our study not only tested the accuracy of the imaging diagnosis of ECS and interobserver agreement between 2 reviewers (A.H.A. and P.A.H.) but also examined the interobserver agreement between the original report and the subsequent blinded review looking specifically for ECS. It was not surprising that the reviewers detected a few more cases of ECS at the expense of more false-positive results. In other words, reviewers became more sensitive ( $67 \%$ compared with $46 \%$ in the original report) but slightly less specific (88\% compared with $95 \%$ in the original report). Overall, both the review and the original report had high specificities for ruling in macroscopic ECS. Interobserver agreement between an original report and a subsequent blinded review has not been evaluated often, but it has important implications for practical use. We have shown that in a closed head and neck radiology group with a focus on staging head and neck cancers, interobserver agreement and specificity for ECS are strong. Our clinicians prefer a high specificity before recommending chemoradiation over transoral robotic surgery.

A very careful search for ill-defined node borders is key for identifying subtle ECS on imaging. We found that imaging specificity is high when fat stranding and ill-defined borders are identified. In these cases, the clinicians can rely on radiographically determined ECS to make treatment decisions (Fig 1A). When these specific imaging signs are absent, however, pECS is still present in nearly $50 \%$ of the cases, and therefore imaging cannot be used to exclude ECS (Fig 2). 


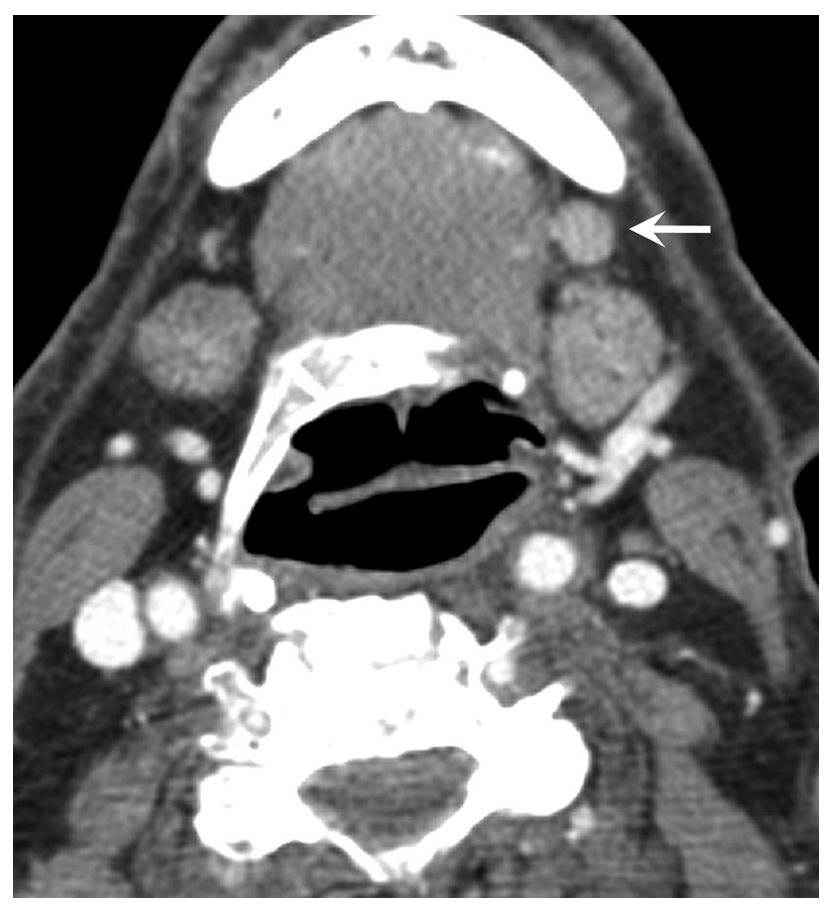

FIG 2. A left 1-cm level IB lymph node (arrow) with abnormal FDG uptake and rounded morphology. Although it was interpreted from contrast-enhanced CT as a metastatic node, it was thought to be negative for ECS. This was a false-negative result, because ECS was found in the final pathologic examination.

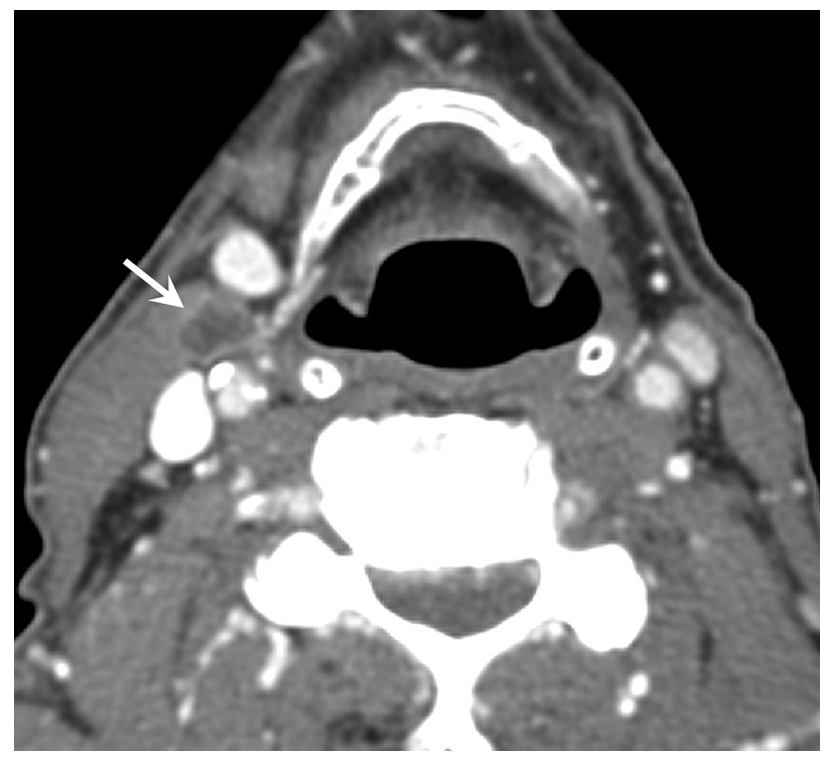

FIG 3. A right 1.2-cm level IIA lymph node (arrow) in a patient with OCSCC. Necrosis was reported and ECS was diagnosed from imaging. This was a true-positive result that was confirmed with pathologic examination.

Lymph node necrosis was the most important radiologic factor associated with radiographically determined ECS even when there was no irregularity of borders or stranding (Fig 3). One study reported a strong correlation between ECS and necrosis with $95 \%$ sensitivity and $85 \%$ specificity. ${ }^{15}$ A recent study by Joo et $\mathrm{al}^{21}$ which involved PET/CT for the identification of ECS, showed that fluorodeoxyglucose uptake could elevate sensitivity and specificity for the detection of ECS above those of contrast- enhanced CT or MR imaging alone. The study showed that maximum standardized uptake values were significantly correlated with ECS (standardized uptake value detection cutoff, 2.25 on receiver operating characteristic curve demonstration; sensitivity and specificity, $85 \%$ and $88 \%$, respectively).

Limitations of this study include its retrospective nature. We used this cohort of patients with OCSCC to examine radiographic accuracy for diagnosing ECS and to extrapolate the diagnostic test characteristics to make treatment decisions for patients with OPSCC. The pathologic pattern of ECS may differ between these subsites, and therefore results of the imaging accuracy evaluation may have been affected. Second, our finding that lymph node necrosis was significantly correlated with ECS may be less reliable in a population of patients with oropharyngeal cancer because of the distinct pattern of cystic nodes in this population. Finally, the importance of both microscopic and macroscopic pECS was first appreciated in the era when oropharyngeal cancers were largely caused by tobacco and alcohol abuse, and as the virally induced oropharyngeal cancers are better understood, the prognostic effects of ECS may not be confirmed. ${ }^{4}$ At this time, however, pretreatment imaging profoundly influences the management of patients with oropharyngeal cancer, and accuracy remains of paramount importance.

\section{CONCLUSIONS}

Recent management trends for OPSCC rely on pretreatment imaging diagnosis of ECS. We used a cohort of patients with OCSCC with pathologically proven or excluded ECS to conclude that though contrast-enhanced CT has high specificity for the detection of ECS, its sensitivity for the detection of ECS remains low. Lymph node necrosis seen on contrast-enhanced CT was significantly correlated with pECS and was the most accurate radiologic criterion of those investigated. Stranding and gross invasion were marginally significant as independent factors correlated with pECS.

Disclosures: Jonathan Beitler-RELATED: Grant: Georgia Cancer Coalition Distinguished Scholar*; UNRELATED: Employment: Connecticut National Guard; Payment for Lectures (including service on speakers bureaus): ASTRO, Head and Neck Cancer Symposium; Payment for Development of Educational Presentations: Oakstone Medical Publishing (Practical Reviews in Cancer Management). Amanda CoreyUNRELATED: Payment for Lectures (including service on speakers bureaus): Osler Institute, Comments: Did an ear, nose, and throat review course lecture to ear, nose, and throat specialists preparing for board examinations. Patricia HudginsUNRELATED: Royalties: Amirsys, Comments: Medical education company. *Money paid to the institution.

\section{REFERENCES}

1. Kokemueller H, Rana M, Rublack J, et al. The Hannover experience: surgical treatment of tongue cancer-a clinical retrospective evaluation over a 30 years period. Head Neck Oncol 2011;3:27 CrossRef Medline

2. Jan JC, Hsu WH, Liu SA, et al. Prognostic factors in patients with buccal squamous cell carcinoma: 10-year experience. J Oral Maxillofac Surg 2011;69:396-404 CrossRef Medline

3. Shaw RJ, Lowe D, Woolgar JA, et al. Extracapsular spread in oral squamous cell carcinoma. Head Neck 2010;32:714-22 CrossRef Medline

4. Maxwell JH, Ferris RL, Gooding W, et al. Extracapsular spread in head and neck carcinoma: impact of site and human papillomavirus status. Cancer 2013;119:3302-8 CrossRef Medline

5. Kann BH, Buckstein M, Carpenter TJ, et al. Radiographic extracapsular 
extension and treatment outcomes in locally advanced oropharyngeal carcinoma. Head Neck 2014;36:1689-94 CrossRef Medline

6. O'Sullivan B, Huang SH, Siu LL, et al. Deintensification candidate subgroups in human papillomavirus-related oropharyngeal cancer according to minimal risk of distant metastasis. J Clin Oncol 2013; 31:543-50 CrossRef Medline

7. Adelstein DJ, Ridge JA, Brizel DM, et al. Transoral resection of pharyngeal cancer: summary of a National Cancer Institute Head and Neck Cancer Steering Committee Clinical Trials Planning Meeting, November 6-7, 2011, Arlington, Virginia. Head Neck 2012;34:1681703 CrossRef Medline

8. Bernier J, Cooper JS, Pajak TF, et al. Defining risk levels in locally advanced head and neck cancers: a comparative analysis of concurrent postoperative radiation plus chemotherapy trials of the EORTC (\#22931) and RTOG (\# 9501). Head Neck 2005;27:843-50 CrossRef Medline

9. Steinkamp HJ, Beck A, Werk M, et al. Extracapsular spread of cervical lymph node metastases: diagnostic value of magnetic resonance imaging [in German]. Rofo 2002;174:50-55 CrossRef Medline

10. Steinkamp HJ, van der Hoeck E, Böck JC, et al. The extracapsular spread of cervical lymph node metastases: the diagnostic value of computed tomography [in German]. Rofo 1999;170:457-62 CrossRef Medline

11. Carvalho P, Baldwin D, Carter R, et al. Accuracy of CT in detecting squamous carcinoma metastases in cervical lymph nodes. Clin $\mathrm{Ra}$ diol 1991;44:79-81 CrossRef Medline

12. Yousem DM, Som PM, Hackney DB, et al. Central nodal necrosis and extracapsular neoplastic spread in cervical lymph nodes: MR imaging versus CT. Radiology 1992;182:753-59 CrossRef Medline

13. King AD, Tse GM, Yuen EH, et al. Comparison of CT and MR imag- ing for the detection of extranodal neoplastic spread in metastatic neck nodes. Eur J Radiol 2004;52:264-70 CrossRef Medline

14. Souter MA, Allison RS, Clarkson JH, et al. Sensitivity and specificity of computed tomography for detection of extranodal spread from metastatic head and neck squamous cell carcinoma. J Laryngol Otol 2009;123:778-82 CrossRef Medline

15. Zoumalan RA, Kleinberger AJ, Morris LG, et al. Lymph node central necrosis on computed tomography as predictor of extracapsular spread in metastatic head and neck squamous cell carcinoma: pilot study. J Laryngol Otol 2010;124:1284-88 CrossRef Medline

16. Saindane AM. Pitfalls in the staging of cervical lymph node metastasis. Neuroimaging Clin N Am 2013;23:147-66 CrossRef Medline

17. Ang KK, Harris J, Wheeler R, et al. Human papillomavirus and survival of patients with oropharyngeal cancer. N Engl J Med 2010;363: 24-35 CrossRef Medline

18. Yokota $\mathrm{T}$, Onitsuka $\mathrm{T}$, Kusafuka $\mathrm{K}$, et al. Is postoperative adjuvant chemoradiotherapy necessary for high-risk oropharyngeal squamous cell carcinoma? Int J Clin Oncol 2014;19:38-44 CrossRef Medline

19. Prabhu RS, Magliocca KR, Hanasoge S, et al. Accuracy of computed tomography for predicting pathologic nodal extracapsular extension in patients with head-and-neck cancer undergoing initial surgical resection. Int J Radiat Oncol Biol Phys 2014;88:122-29 CrossRef Medline

20. Chai RL, Rath TJ, Johnson JT, et al. Accuracy of computed tomography in the prediction of extracapsular spread of lymph node metastases in squamous cell carcinoma of the head and neck. JAMA Otolaryngol Head Neck Surg 2013;139:1187-94 CrossRef Medline

21. Joo YH, Yoo IR, Cho KJ, et al. Extracapsular spread and FDG PET/CT correlations in oral squamous cell carcinoma. Int $J$ Oral Maxillofac Surg 2013;42:158-63 CrossRef Medline 